\title{
Ces étranges machines extrême-occidentales
}

Le Qi qi Tu Shuo, une invention livresque

Strange Machines of the Far East. The Qi qi Tu Shuo, a literary invention

\section{Georges Métailié et Hélène Vérin}

\section{(2) OpenEdition}

Journals

Édition électronique

URL : http://journals.openedition.org/artefact/1874

DOI : 10.4000/artefact.1874

ISSN : 2606-9245

Éditeur :

Association Artefact. Techniques histoire et sciences humaines, Presses universitaires du Midi

Édition imprimée

Date de publication : 6 décembre 2018

Pagination : $15-47$

ISBN : 978-2-8107-0595-5

ISSN : 2273-0753

Référence électronique

Georges Métailié et Hélène Vérin, «Ces étranges machines extrême-occidentales », Artefact [En ligne],

8 | 2018, mis en ligne le 21 juin 2019, consulté le 28 novembre 2020. URL : http://

journals.openedition.org/artefact/1874 ; DOI : https://doi.org/10.4000/artefact.1874

\section{cc) (†)}

Artefact, Techniques, histoire et sciences humaines est mise à disposition selon les termes de la Licence Creative Commons Attribution - Pas d'Utilisation Commerciale - Pas de Modification 4.0 International. 


\title{
Ces étranges machines extrême-occidentales
}

\author{
Le Qi qi Tu Shuo, une invention livresque
}

\section{Georges Métailié et Hélène Vérin}

\section{Résumé}

Fruit d'une collaboration entre un lettré chinois converti au christianisme Wang Zheng (1571-1644) et un missionnaire jésuite Johann Schreck, dit Terrentius (1576-1630) le livre intitulé « Essentiel des explications et représentations de merveilleuses machines d'Extrême-Occident » Yuanxi qiqi tushuo luzui, fut publié en 1627. Il rassemble dans un même ouvrage des données théoriques de la science des poids, d'analyse et de calcul de leurs applications dans les machines simples et de présentation de divers modèles de machines. Ce rassemblement constitue une approche inédite qui n'a pas sa pareille en Occident. C'est cette innovation que nous proposons d'interroger.

\section{Mots-clés}

traité de mécanique, théâtres de machines, lettrés, missionnaires, sinisation, terminologie, graphisme

99 Georges Métailié et Hélène Vérin, « Ces étranges machines extrême-occidentales 》, Artefact, 8, 2018, p. 15-47. 


\section{Strange Machines of the Far East The Qi qi Tu Shuo, a literary invention}

\section{Abstract}

The book "Collected Diagrams and Explanations of the Wonderful Machines of the Far West" Yuanxi qiqi tushuo luzui, was published in 1627, being a joint work by the Chinese Christian scholar Wang Zheng (1571-1644) and the Jesuit missionary Johan Schreck, also known as Terrentius (1576-1630). A compilation of theoretical data concerning the science of weight, with analysis and calculations for its uses in simple machines, it also includes various instructional diagrams of machines. This approach was unprecedented and knew no equivalent in the West. Our inquiry addresses this innovative form of scholarship.

\section{Keywords}

treatise of mechanics, mechanical implements, scholar-officials, missionary, sinicisation, terminology, graphics

\section{Note des auteurs}

Cet article a fait l'objet d'une présentation au $x x^{\mathrm{e}}$ colloque international d'Études humanistes, Tours, 29 juin-1 ${ }^{\text {er }}$ juillet 2005 qui n’a pas donné lieu à publication. Le manuscrit a circulé depuis parmi des collègues ou des étudiants qui nous l'avaient demandé. Nous avons ici mis à jour les références en indiquant des articles parus depuis sur le sujet. 


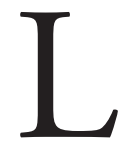

e "Yuanxi Qiqi Tushuo Luzui », QQTS, « Essentiel des explications et représentations de merveilleuses machines d'Extrême-Occident » est le produit d'une collaboration entre un lettré chinois, Wang Zheng et un jésuite allemand, Johann Schreck dit Terrens ou Terrentius ${ }^{1}$. L'histoire de la co-production du QQTS que Wang Zheng expose dans sa préface commence à être mieux connue ${ }^{2}$. Après avoir été publié en 1627 à Yangzhou, l'ouvrage a fait l'objet de plusieurs rééditions remaniées au cours des deux siècles suivants ${ }^{3}$.

Le troisième chapitre du QQTS a été inclus dans le Gujin Tushu Jicheng (GJTSJC, Collection des textes et illustrations anciens et modernes) qui fut imprimé pour la première fois en 1726, puis l'ouvrage entier fut édité dans le Si Ku Quan Shu (SKQS, Collection complète des quatre trésors), qui fut compilé durant le règne de l'empereur Qianlong (1736-1795). Au $\mathrm{XIX}^{\mathrm{e}}$ siècle, il connut plusieurs réimpressions qui introduisirent quelques modifications. Ainsi dans l'édition du QQTS comprise dans le Shoushange congshu (Collection du Pavillon Shoushan) (1844) les lettres romaines de renvoi des figures de machines aux textes explicatifs furent changées en caractères chinois. Une édition de 1877 changea le nom même de l'ouvrage en Ji Qi Tu Shuo (Diagrammes et explications de machines). Cette fortune livresque du QQTS qui témoigne d'une curiosité pour les machines occidentales, n’aurait guère eu d'influence, selon les historiens, sur la mécanique théorique et pratique de la Chine traditionnelle.

Le livre, après une introduction importante comprend trois grands chapitres : le premier, Zhong Jie, " explication des poids " consacre ses $61 \mathrm{sec}-$ tions à l'exposé d'éléments de mécanique théorique et de la «science des

1. Voir la réédition récente annotée, fondée sur une édition chinoise de 1830, par ZHang Baichun, Tian Miao, Matthias Schemmel, Jürgen Renn, Peter Damerow, Chuan bo yu hui tong : "Qi qi tu shuo" yan jiu yu jiao zhu: Transmission and integration - Qiqi Tushuo (illustrations and descriptions of extraordinary devices): New research and annotated edition, Nanjing, Jiangsu ke xue ji shu chu ban she, 2008, vol. 2. Les auteurs de cet ouvrage préparent actuellement (2018) une traduction complète du QQTS en anglais accompagnée de commentaires.

2. En particulier, voir KIM Yung Sik, "A Philosophy of Machines and Mechanics in seventeenthCentury China: Wang Zheng's Characterization and Justification of the Study of Machines and Mechanics in the Qiqi tushuo ", EASTM 31, 2010, p. 64-95. Voir aussi ZHANG Baichun et al. (éd.), op. cit., vol 1, p. 50-82.

3. Zhang Baichun, Tian Miao et Liu Qiang, (en Chinois) «Évolution des éditions du Yuanxi Qiqi Tushuo Luzui et du Xinzhi zhu qi tu (Illustrations de plusieurs machines nouvellement construites). The Chinese Journal for the History of Science and Technology = Zhongguo Kejishi Zazhi, 2006, 27(2), p. 115-136, et p. 182-223, in ZHANG et al. 2008, op. cit. 
poids " : centre de gravité, centre géométrique, poids spécifique, étude des corps flottants... Le chapitre deux, Qi jie, "explication des instruments " comprend 92 sections et examine les principes et le calcul des machines simples : balance, balance romaine, levier, poulie, roue, vis, plan incliné. Le troisième chapitre présente 54 modèles de machines selon le mode d'exposition propre aux théâtres de machines occidentaux d'où ils sont tirés : une planche en pleine page, accompagnée, en regard, d'une " explication de la figure ".

Comment ce livre a-t-il vu le jour?

\section{Un lettré s'empare de livres étrangers pour produire un livre chinois}

Johann Schreck (1576-1630) naît en Suisse alémanique. Très tôt il développe un grand intérêt pour divers domaines scientifiques, mathématiques, médecine et botanique, astronomie. En 1603 il va étudier la médecine à l'université de Padoue où Galilée enseignait depuis 1592 et ce fut l'occasion de leurs premières rencontres. Sa réputation scientifique devint rapidement assez grande pour que le Prince Cesi, fondateur de l'Academia dei Lincei, à Rome, haut lieu de la philosophie naturelle et des premières expérimentations de la physique, lui confie en 1610 la traduction latine du grand ouvrage de Francisco Hernandez sur l'histoire naturelle de la Nouvelle Espagne ${ }^{4}$. Lenjeu était de connaître les plantes - en particulier médicinales - utilisées par les Indiens. En 1611, il est admis, en même temps que Galilée, à cette Académie. L'année même de son entrée, Schreck, dorénavant Terrentius, décide de rejoindre l'Ordre des Jésuites et il commence son noviciat le $1^{\text {er }}$ novembre. De ce fait, tout en restant en contact avec divers académiciens, il se retire officiellement de l'Academia, et c'est en 1616 qu'il intègre définitivement l'Ordre. Cette année-là

\footnotetext{
4. Quatro libro de la naturaleza y virtudes de las plantas y animales que estan receivedos en el uso de medicina en Nueva Espana... que el Doctor Francisco Hernandez escrivio en lengua latina, traduzido por Fr Francisco Ximenes, Mexico, Diego Lopez Calvado, 1615. Cette traduction plus complète et commentée à laquelle contribuèrent Johannes Faber et Fabius Colonna, membres de l'Academia de lincei, parut entre 1628 et 1651. Rerum medicarum Novae Hispaniae thesaurus seu Plantarum animalium mineralium Mexicanorum historia ex Francisci Hernandez... A Nardo Antonio Reccho... Collecta ac in ordinem digesta, A Joanne Terrentio Lynceo... Notis illustrata, Romae, Ex typographeio Vitalis Mascardi.
} 
Nicolas Trigault, fondateur avec Matteo Ricci de la mission jésuite en Chine, rentre en Europe pour recruter d'autres jésuites scientifiques et chercher auprès des cours européennes des financements, acheter livres et instruments scientifiques destinés à la mission en Chine. Schreck se joint à lui ainsi que les Pères Giacomo Rho et Johann Adam Schall von Bell. Dès 1618 le nouveau groupe de jésuites quitte Lisbonne pour la Chine en passant par Goa, le Bengale, Malaca, Sumatra, l'Indochine. Terrentius arrive finalement à Macao où il va rester deux ans qu'il met à profit pour commencer l'apprentissage du chinois et surtout continuer de collecter en naturaliste plantes inconnues, pierres rares, animaux de toutes espèces, avec l'intention de publier un "Plinius Indicus » comparable au " Mexicanus » d'Hernandez. Cet ouvrage qu'il continua durant son séjour en Chine ne fut jamais terminé. Le manuscrit a depuis disparu. C’est à Pékin, qu'il atteint à la fin de 1623, qu'il rencontre Wang Zheng, converti qui est son élève en religion et avec qui il continue son apprentissage du chinois. Dans le même temps il poursuit ses travaux astronomiques, entretient une nombreuse correspondance avec des savants européens, dont Galilée et Kepler, et s'engage dans son projet de traduction en chinois de livres scientifiques rapportés d'Europe ${ }^{5}$.

Wang Zheng (1571-1644) de la province du Shanxi, était le fils d'un mathématicien, théoricien du confucianisme. Il réussit les examens impériaux et eut une carrière de fonctionnaire. Il commença très jeune à s'intéresser aux machines et à la mécanique pratique. L’idée de cet ouvrage lui était venue, dit-il, à la suite de son contact avec les jésuites missionnaires et la connaissance qu'il put acquérir des ouvrages européens qu'ils avaient introduits en Chine et des premiers écrits en chinois qu'ils avaient diffusés ${ }^{6}$. En particulier, le Zhi Fang Wai Ji d'Aleni (ZFWJ, Enregistrement des endroits extérieurs à la juridiction du bureau de la géographie, 1623) ${ }^{7}$. Ainsi la convergence entre son intérêt pour les machines, son confucianisme qui

\footnotetext{
5. Cette brève note biographique s'appuie sur Giuseppe Gabrieli, "Giovanni Schreck Linceo, Gesuita e Missionario in Cina e le sue Lettere dell'Asia ", Rendiconti della classe di scienza morale, storiche e filologiche. Ser. II. Roma, 12, 1936, p. 462-514. La liste des ouvrages traduits se trouve p. 477-479. Autre édition in Rome, Royal National Academy of the Lincei, 1937.

6. Nous avions utilisé une édition chinoise du XIX siècle, non datée, fondée sur l'édition figurant dans le Si Ku Quan Shu. Nous indiquons en premier les pages en référence à cette édition et, en second, celles qui correspondent à celles de la réédition récente par ZHANG Baichun et al. op. cit., vol. 2. Préface $x u$, 2a.// Zhang et al., op. cit., vol. 2, p. 17.

7. Préface $x u, 1 \mathrm{~b} . / /$ Zhang et al., op. cit., vol. 2, p. 16.
} 
l'incline à travailler au bien public et l'opportunité que lui offrent ses relations avec les jésuites vont le conduire à l'idée d'une diffusion chinoise de modèles de machines occidentales qui pourraient être utiles à ses concitoyens. L'ambition déclarée est d'atteindre un public élargi, dans des vues qui se présentent volontiers comme altruistes. Un tel propos suppose toute une série de conditions.

\section{Les attendus socio-religieux}

La première condition de réussite du projet est qu'il soit acceptable socialement. Wang Zheng consacre un long passage de sa préface à cette question. Il y fait état des réticences que cette publication rencontra sous la forme d'une narration, celle de ses discussions avec des « visiteurs bienveillants à son égard » qui seraient venus le mettre en garde contre l'inconvenance et les dangers d'une telle production. D'abord, il était inconvenant pour un lettré de s'intéresser à un sujet trivial qui relève des qualifications d'un artisan. Un homme d'honneur qui consacre ses pensées à des ustensiles remet en cause sa condition et donc l'ordre social.

Le deuxième argument des visiteurs concerne la collaboration de Wang Zheng avec les missionnaires jésuites. Ces hommes, venus de lointaines contrées désolées ne sont pas de même rang qu'un fonctionnaire impérial qui se met néanmoins en position d'être leur disciple.

La réponse de Wang Zheng au premier reproche de ses visiteurs est d'insister sur la valeur de l'objectif qu'il s'est ainsi donné : être bénéfique à la société. À partir de là, les moyens qu'il utilise, pourvu qu'ils n'aillent pas contre le Ciel, sont en eux-mêmes indifférents : qu'il s'agisse de la valeur intellectuelle des connaissances mises en œuvre, de la nation des hommes qui les ont apportées ou de la qualité triviale ou pas de leur contenu. L'essentiel est cette finalité qui est conforme aux enseignements de Confucius. À l'appui de quoi Wang Zheng décline une suite d'affirmations de facture proverbiale $^{8}$ : "Rencontrer des personnes extraordinaires est rare. On entend rarement un savoir unique. Poursuivre ensemble après une rencontre est le plus difficile. Le temps n'attend pas. Découvrir ces merveilles et ne pas les noter pour les transmettre est pour moi impossible ».

8. Préface $x$ u, 4a,b. // Zhang et al., op. cit., vol. 2, p. 22. 
Wang Zheng inscrit alors cet ouvrage dans la suite de ses écrits : il s'est d'abord efforcé de chercher des choses qui peuvent aider "les yeux et les oreilles " des hommes ${ }^{9}$; ici il s'agit d'aider leurs pieds et leurs mains. Pourquoi s'en inquiéter? Suit un long passage où il souligne les avantages que la Chine peut tirer de ce que les catholiques ont apporté et aussi l'appui impérial dont ils ont déjà disposé. Néanmoins, il est remarquable qu'à aucun moment Wang Zheng n'évoque sa conversion au christianisme dont on peut penser qu'elle n'est pas étrangère à son attitude. En effet, Si l'altruisme est bien l'une des vertus cardinales du confucianisme, l'interprétation généreuse qu'il en fait et qui choque ses égaux chinois, doit sans doute beaucoup à l'une des vertus cardinales du christianisme, la charité ${ }^{10}$.

Un autre visiteur est alors présenté, qui aurait accepté la valeur des ambitions de Wang Zheng mais l'accuse d'avoir mal dirigé ses efforts : pourquoi aider les yeux, les oreilles, les mains et les pieds des hommes, et non leur esprit ? Pourquoi ne pas s'être attaché à traduire des ouvrages relevant de connaissances supérieures?

Selon Wang Zheng, l'étude et la traduction de telles connaissances relèveraient davantage des compétences de ceux qui se consacrent officiellement à ces études : les maîtres de l'Académie impériale et de la Bibliothèque impériale. Coup double : il va dans le sens des jésuites qui se servent des sciences et des techniques pour s'introduire dans la haute société chinoise, et dénonce l'idée qu'il serait leur simple disciple en se situant par rapport aux lettrés de l'Académie.

La critique néanmoins se poursuivit et poussa plus loin encore son attaque en dénonçant, dans un tel projet, porté par un lettré fonctionnaire, la remise en cause de l'ordre du monde et de la doctrine de la régulation

9. Wang Zheng évoque ici le Xiru ermu zi (1626) (Aide dans la lecture et l'écoute des lettrés d'Occident) par Nicolas TRIGault. Sur ces problèmes de transcriptions du chinois ainsi que sur les travaux des missionnaires européens en Chine, voir Nicolas Standaert (dir.), Handbook of Christianity in China, Volume One : 635-1800, Leyde/Boston, Brill, 2001. Sur les réactions chinoises au christianisme, voir Jacques GeRnet, "Langage et pensée », Chine et christianisme. Action et réaction, Paris, Gallimard, 1982, p. 322-333. Voir aussi, du même auteur, "Logique discursive et logique combinatoire ", in Études chinoises, 2003, n 22 , p. 19-46.

10. C'est également l'argument avancé par les auteurs de théâtres de machines utilisés dans le QQTS, en particulier par Ramelli. Luisa Dolza et Hélène VéRIN, « Figurer la mécanique : l'énigme des théâtres de machines de la Renaissance ", Revue d'histoire moderne et contemporaine, n 51-2, avril-juin 2004, p. 7-31. 
Zhong Yong ${ }^{11}$. Il faut attendre 1829 avec la préface de Zhang Pengfen pour voir se mettre en place une histoire de Wang Zheng à caractère mythique qui en fait un héros mais aussi un " martyr " dévoué à la cause du bien commun.

\section{Précédents et sources}

Dans sa préface Wang Zheng fait état des précédents chinois bien antérieurs à l'arrivée des jésuites et qui avaient été au point de départ de son intérêt pour les mécaniques. Il cite la sphère armillaire et la boussole qu'il avait, dit-il, construites lui aussi et signale que les techniques utilisées pour leur fabrication n'ont pas été transmises. Au contraire, le fameux muniu liuma (bœuf en bois, cheval courant) a perduré ${ }^{12}$. Lui-même est l'auteur de plusieurs dispositifs : Hongxi (une pompe actionnée par un cylindre simple), heyin (un échangeur à canalisations rayonnées), lunhu (une horloge complexe), daigeng (une charrue-treuil actionnée par un cable), zizhuanmo (un moulin actionné par un poids) et zixingche (un quadricycle auto-mobile). Ces machines, dit-il, ont fonctionné et ont été appréciées ${ }^{13}$.

Selon ses dires, c'est en prenant connaissance de l'ouvrage d'Aleni, le $Z$ hi Fang Wai Ji, que son émerveillement devant les machines occidentales l'a conduit à l'idée d'en faire un recueil chinois. Il cite deux machines d'élévation d'eau pour l'approvisionnement urbain : celle de Tolède et celle qui

11. Voir François Jullien (dir.), Zhong Yong ou La Régulation à usage ordinaire, Paris, Imprimerie nationale, 1993.

12. Il s'agit à l'origine d'engins pour transporter les vivres dont l'invention est attribuée à Zhuge Liang (181-234). On ne sait pas exactement de quoi il s'agissait. C'est, en fait, le terme « bœuf de bois » qui a perduré et a été repris par la suite pour désigner une charrue tractée à l'aide d'une corde reliée à un treuil animé par deux hommes, d'un côté du champ à labourer et passant, de l'autre côté par une poulie. Guidée par un troisième homme, la charrue pouvait ainsi avancer ou reculer. Wang Zheng en donne un exemple européen, tiré de Jacques Besson (chap. 3, 54b-55a ; Zhang et al., op. cit., vol. 2, p. 180-181) et propose son propre modèle simplifié dans son traité Xinzhi zhu qi tu "Représentations de machines nouvellement fabriquées », joint au QQTS (p. 15b / Zhang et al., op. cit., vol. 2, p. 208). Dans les deux cas, le terme «bœuf de bois » n'est pas repris ; il s'agit de machines pour "suppléer au labour", daigeng.

13. Sur cette question, voir Ladislao Retr, "The Double-Acting Principle in East and West", Technology and Culture, vol. 11, $\mathrm{n}^{\circ}$ 2, avril 1970, p. 178-200. Bert S. HALL, "A revolving bookcase by Agostino Ramelli ", Technology and Culture, vol 11, n 3, juillet 1970, p. 395-396. Dans le Xinzhi zhu qi tu (Illustrations de plusieurs machines nouvellement construites) ajouté au QQTS, dont Wang Zheng est l'auteur, on peut voir plusieurs de ces machines. Voir Zhang et al., op. cit., vol. 2, p. 194-214. 
est constituée de vis d'Archimède mises en série, probablement, la machine d'Augsbourg dessinée et décrite par Cardan dans son de Subtilitate ${ }^{14}$. Il cite également deux machines mythiques d'Archimède : celle qui lui aurait permis de porter un vaisseau jusqu'à la mer et la fameuse sphère armillaire de verre aux mouvements automatiques.

Pour conclure, Wang Zheng n'est pas en terre inconnue quand il aborde les descriptions de machines. Si le QQTS est bien un ouvrage unique et original, d'autres textes chinois, en particulier sur l'agriculture, montraient et décrivaient non seulement des outils mais aussi des machines, à vanner et à moudre les grains, à élever de l'eau, des forges, des métiers à tisser... et ceci dès le $\mathrm{XIV}^{\mathrm{e}}$ siècle, tandis que divers textes des $\mathrm{XI}^{\mathrm{e}}$ et $\mathrm{XII}^{\mathrm{e}}$ siècles décrivent des tours-horloges avec globe céleste et sphère armillaire. Un grand ouvrage encyclopédique San cai tu hui de Wang Qi et Wang Siyi, publié en 1609, soit dix-huit ans avant notre livre, reprend nombre des illustrations commentées de ces machines et y ajoute, par exemple, des machines de guerre ${ }^{15}$. À certains égards, il s'agissait pour Wang Zheng de compléter ces recueils par de nouveaux modèles et de le faire selon un mode de présentation où se rejoignent une tradition chinoise et une invention de la Renaissance, les théâtres de machines.

À la suite de la préface de Wang Zheng se trouve une liste des sources alors disponibles en Chine ; elle rassemble les titres d'ouvrages en chinois traduits ou rédigés par des missionnaires et des lettrés convertis entre 1596 et 1623 :

- Tian zhu shi yi (Dei Vera Ratio), de Matteo Ricci (1596);

- Ii he yuan ben (Éléments de géométrie) (Clavius, Euclidis Elementorum libri XV, Rome), Matteo Ricci et Xu Guangqi, 1607 ;

- Gou gu fa yi (Le sens de la méthode gougu), serait le Gou gu yi, (un traité des triangles rectangles) de Xu Guangqi, 1608 ;

- Ji ren shi pian (Biographies de Dix personnes spéciales), Matteo Ricci, vers 1608 ;

14. Girolamo Cardano, De subtilitate, 1663, vol 1, p. 367 ; édition critique Elio Nenci, Milan, F. Angeli, 2004.

15. Voir Wang Qi, Wang Siyi, San Cai Tu Hui (Encyclopédie universelle) (1609), Shanghai, Guji chubanshe, 1988, 3 vol., vol. 2, p. 1186-1190 ; p. 1200-1207. Images et diagrammes ont joué un rôle important dans le développement de la pensée technique en Chine. Voir Francesca BraY, Vera Dorofeeva-Lichtman, Georges Métailié (dir.), The Warp and the Weft: Graphics and Text in the Production of Technical Knowledge in China, Leyde, Brill, 2007. 
- Tai xi shui fa (Méthodes hydrauliques d'Extrême-Occident), Sabatin de Ursis, 1612 ;

- Yuan rong jiao yi (Le sens de [figures] comparées inscrites dans un cercle) de Matteo Ricci et Li Zhizao, 1614 ;

- Tong wen suan zhi (Règles d'arithmétique commune aux Cultures :

C. Clavius, Epitome Arithmeticae Practicae, Rome, 1583), de Matteo Ricci et Li Zhizao, 1614 ;

- Qi ke (Sur la morale catholique), Diego de Pantoja, 1614;

- Tian wen lüe (Questions au sujet du ciel), Manuel Dias, 1615;

- Jian ping yi shuo (Explication de la sphère armillaire), Sabatino de Ursis, 1616 ;

- Zi ming zhong shuo (Explication de l'horloge à sonnerie automatique) ;

- Zhi fang wai ji (Enregistrement des lieux extérieurs à la juridiction de l'Office de géographie), Giulio Aleni, 1623 ;

- Xi xue huo wen (Enquête sur les savoirs occidentaux);

- Xi xue fan (Introduction générale aux savoirs occidentaux), Giulio Aleni, 1623 ;

- Wang yuan jing shuo (Explication du Télescope, 1626).

Si l'on considère les sources directement utilisées par Wang Zheng, on peut voir qu'il annonce au premier chapitre de son livre que « les auteurs les plus célèbres sur la mécanique sont, comme théoriciens Wei Touo et Simon et comme praticiens, King tien et Lamelli ${ }^{16}$ ». H. Verhaeren dans son article de 1947 "Wang Tcheng et la mécanique » est le premier qui s'efforce de les identifier : "Je ne crois pas me tromper, affirme-t-il, en supposant que Weitouo est une translittération raccourcie de Vitruve, que Simon désigne Stévin [...] que King tien est la traduction chinoise du nom d'Agricola et que Lameli figure le nom italien de Ramelli ${ }^{17}$ ». Verhaeren s'appuyait sur le fait que les ouvrages de ces auteurs se trouvaient dans la bibliothèque du Pét'ang dont il dressa le catalogue ${ }^{18}$. Si nous nous tenons aux seuls "praticiens ", dont les machines sont présentées au chapitre III du QQTS, nous pouvons identifier précisément cinq ouvrages :

16. Juan 1, 4b // ZHANG et al., op. cit., vol. 2, p. 40.

17. Hubert Verhaeren, "Wang Tcheng et la mécanique ", Le Bulletin catholique de Pékin, $34^{\mathrm{e}}$ année, 1947, p. 185-186.

18. Hubert Verhaeren, Catalogue de la Bibliothèque du Pé-t’ang, Beijing, Imprimerie des Lazaristes, 1949 [reprint Paris, Les Belles-Lettres, 1969]. 
- Jacques Besson, Théatre des instruments mathématiques et méchaniques de Jacques Besson, avec l'interpretation des figures d'iceluy, par Francois Béroald, Lyon, B. Vincent, 1578 ;

- Agostino Ramelli, Le Diverse et artificiose machine del capitano Agostino Ramelli dal ponte della Tresia... Nelle quali si contengono varii et industriosi movimenti... composé en langue italienne et françoise, Paris, chez l'auteur, $1588^{19}$;

- Fausto Veranzio, Machinae Novae, cum declaratione latina italica, hispanica, gallica et germanica, Venise, $1595^{20}$;

- Heinrich Zeising, Theatri machinarum erster, $1^{\text {re }}$ édition, 1607. L'œuvre complète comprend six parties : les trois premières dues à l'éditeur Henning Gross, les dernières à Hieronymus Megiser. Leipzig, H. Grossen le jeune, $1612-1614^{21}$;

- Vittorio Zonca, Novo teatro di machine et edificii per varie et sicure operationi, con le loro figure tagliate in rame é la dichiarazione e dimostrazione di ciascuna, opera necessaria ad architetti et a quelli che di tale studio si dilettano, Padoue, P. Bertelli, $1607^{22}$.

La distribution des machines présentées dans le chapitre III obéit au classement suivant ${ }^{23}$ :

\begin{tabular}{|cl|rl|}
\hline \multicolumn{2}{|l|}{ Lever des poids } & \multicolumn{2}{|c|}{ Moulins à scier le bois } \\
Figure 1 & balance romaine chinoise & figure 1 & Zeising, III, 7 \\
2 à 6 & Zeising, I & 2 & Ramelli 136 \\
7 & Besson 35 & 3 & Besson 13 \\
8 & Besson 39 & 4 & Veranzio 44 \\
9 & Ramelli 139 & & \\
10 & -------170 & & \\
11 & $--------~ 74$ & & \\
\hline
\end{tabular}

19. Disponible en ligne : http://cnum.cnam.fr.

20. Disponible en ligne : http://www.europeana.eu/portal/fr.

21. Notons que l'ouvrage de Zeising étant largement une compilation des théâtres de machines précédents, certains modèles présentés peuvent lui avoir été empruntés aux théâtres de machines antérieurs.

22. Disponible en ligne : http://gallica.bnf.fr.

23. Pour une comparaison entre les images originales et leur reproduction dans le QQTS, voir :

ZHANG Baichun et al., 2008, op. cit., vol. 1, p. 126-153. 


\begin{tabular}{|c|c|c|}
\hline \multicolumn{2}{|c|}{ Transporter des poids } & Moulin à scier la pierre \\
\hline Figure 1 & Ramelli 178 & 1 figure $\quad$ Ramelli 134 \\
\hline 2 & ----- 180 & \\
\hline 3 & Besson 30 & \\
\hline 4 & Veranzio 78 & \\
\hline \multicolumn{2}{|c|}{ Élever des poids par rotation } & Marteau à bascule vertical \\
\hline $\begin{array}{r}\text { Figure } 1 \\
2\end{array}$ & $\begin{array}{l}\text { Ramelli } 85 \\
----89\end{array}$ & 1 figure $\quad$ Besson 25 \\
\hline \multicolumn{2}{|l|}{ Élévations d'eau } & \multirow{3}{*}{\begin{tabular}{|l} 
Bibliothèque tournante \\
1 figure $\quad$ Ramelli 188
\end{tabular}} \\
\hline Figure 1 & Ramelli 45 & \\
\hline 2 & ------- 47 & \\
\hline 3 & ------- 61 & \multirow{7}{*}{$\begin{array}{l}\text { Horloge à eau } \\
1 \text { figure Ver }\end{array}$} \\
\hline 4 & -----13 & \\
\hline 5 & $-\cdots-112$ & \\
\hline 6 & Besson 46 & \\
\hline 7 & Zonca 112 & \\
\hline 8 & ------ 110 & \\
\hline 9 & Ramelli 109 & \\
\hline \multicolumn{2}{|l|}{ Moulins à moudre } & \multirow{16}{*}{\begin{tabular}{|l}
\multicolumn{2}{|l}{$\begin{array}{l}\text { Machine à labourer } \\
1 \text { figure }\end{array}$} \\
$\begin{array}{ll}\text { Canons à eau } \\
2 \text { figures }\end{array}$ \\
\end{tabular}} \\
\hline Figure 1 & Ramelli 123 & \\
\hline 2 & ------ 124 & \\
\hline 3 & ----- 128 & \\
\hline 4 & --o-- 130 & \\
\hline 5 & Zonca 88-89 & \\
\hline 6 & Veranzio 23 & \\
\hline 7 & ------- 8 & \\
\hline 8 & ------- 9 & \\
\hline 9 & $-\cdots-10$ & \\
\hline 10 & ------- 10 & \\
\hline 11 & ------ 12 & \\
\hline 12 & ------ 13 & \\
\hline 13 & -------- 22 & \\
\hline 14 & -------11 & \\
\hline 15 & ------- 15 & \\
\hline
\end{tabular}




\section{Le Qi qi Tu Shuo: une invention livresque}

Ce qui intéresse à l'évidence Wang Zheng dans les théâtres de machines européens est l'association texte-image pour transmettre des modèles de machines. Le titre même de son livre: "Choix de figurations graphiques et d'explications " en est l'expression. Nous avons vu que ce mode de transmission de connaissances mécaniques lui est familier. La question est alors de mesurer comment, et jusqu'à quel point, il va lui être possible de remplir les exigences de son projet : mettre ces modèles à la disposition de la Chine. La première difficulté qui concerne ce mode d'exposition par association de textes et de figures est celle des limites du pouvoir informatif du procédé : transmet-il les informations nécessaires et suffisantes pour que chacun des modèles présentés puisse effectivement être réalisé ? Wang Zheng en est persuadé. Or la confiance qu'il accorde aux théâtres de machines est loin d'être partagée en Europe où trois reproches leur sont faits : le premier concerne l'adéquation au principe d'économie qui veut que rien ne soit fait en vain. Il s'agit ici plus précisément de l'économie dans le mode de présentation des modèles. Les théâtres de machines témoignent d'une grande complaisance pour une sorte de virtuosité mécanique ostentatoire qui multiplie à l'infini les modèles et les variations sur des modèles sans permettre au lecteur d'y trouver facilement réponse à ses problèmes. Second reproche : quant à la technique, ce mode de présentation ne fournit pas les moyens de la fabrique. Enfin, quant à la connaissance, ce mode de présentation ne permet pas de rendre raison des effets prétendument obtenus. Ces connaissances ne peuvent s'acquérir que dans des ouvrages ayant la forme de traités. Nous touchons là un point important : quel est ce mode d'exposition ? Que vise-t-il à transmettre ? Ou encore, quel est le régime de savoir qui s'exprime dans la forme "théâtres" de machines ? Il n'est ni celui des déductions de la science des mécaniques, ni celui des codes gestuels et instrumentaux de la fabrique artisanale sur lesquels ils demeurent silencieux. Or ces deux régimes de savoir non seulement sont indispensables à la conception et à l'exécution des modèles proposés, mais encore ils constituent l'ensemble des contraintes et des possibles qui alimentent et règlent les projets du concepteur. 
Placés devant la demande insistante de Wang Zheng de fabriquer un théâtre de machines chinois, comment vont réagir les missionnaires jésuites qui, non seulement doivent être au courant des reproches faits en Europe à ces livres au nom de l'efficacité technique, mais encore, au nom de la science des mécaniques. La réponse est qu'ils vont concevoir un objet inédit en Europe comme en Chine. Si l'on s'en tient à l'aspect purement formel du QQTS on voit qu'il est constitué de trois chapitres dont les deux premiers sont des traités et le troisième un " théâtre ». Or ces deux genres littéraires non seulement ne sont jamais rassemblés dans un même ensemble, comme nous l'indiquions plus haut, mais ils sont l'œuvre de personnages qui n'occupent pas les mêmes places dans l'ordre social des savoirs. Les auteurs de théâtres de machines sont des ingénieurs ou des architectes praticiens qui, lorsqu'ils enseignent les mathématiques, le font en langue vernaculaire et non en latin, et qui doivent leur notoriété à leurs fonctions d'experts auprès d'un prince. Ils attendent de leurs théâtres la reconnaissance de leur capacité à inventer de nouveaux dispositifs, reconnaissance élargie au public des lecteurs. Les auteurs de traités de mécanique, même élémentaires ou appliqués aux machines simples, utilisent une forme discursive à visée pédagogique qui procède de l'abstrait au concret et du simple au complexe, forme qui n'a rien à voir avec celle des théâtres de machines.

Si l'on envisage à présent les insuffisances reprochées aux théâtres de machines du point de vue du contenu des connaissances transmises, on s'aperçoit que l'ouvrage que Johann Schreck va élaborer avec Wang Zheng a l'ambition de pallier les défauts, les manques des théâtres de machines aux niveaux que nous avons relevés : celui de l'économie fonctionnelle non seulement de la forme de l'exposé mais des modèles eux-mêmes ; et surtout, et avant tout, celui des connaissances indispensables pour concevoir des mécanismes opérationnels. C’est l'objet des deux traités préliminaires à quoi s'ajoute au cours même de la présentation des machines, le souci d'indiquer explicitement les contraintes de la fabrique.

\section{Le point de vue de l'économie : la sélection des modèles par Wang Zheng}

À l'ostentation graphique des théâtres de machines se rattache l'infinie variété des modèles trop complexes et trop lourds, trop coûteux aussi. Le QQTS se présente comme une amélioration, puisqu'il s'agit d'une 
" sélection " guidée par un souci d'utilité et d'usages possibles dans son pays. Au nom de l'utilité, Wang Zheng explique dans sa préface qu'il a éliminé les machines curieuses ou d'agrément comme l'oiseau volant ou l'orgue hydraulique ${ }^{24}$. Il a également exclu celles qui mettaient en ouvre trop d'engrenages et de vis sans fin et dont la complexité aurait excédé les capacités des artisans. Enfin, il prend en compte l'estimation du coût de fabrication. Sont donc enregistrées les plus simples et les plus commodes. Le choix n'est pas allé sans difficultés explique-t-il, vu non seulement la multiplicité des modèles, mais encore les variations sur un même principe d'action. Les méthodes, dit-il, y ont beaucoup de variétés et les variations beaucoup de modèles et il donne l'exemple des machines hydrauliques pour lesquelles on peut compter plus de cent dix variantes dont un bon nombre est trop lourd ou trop compliqué. On retrouve donc ici, mot pour mot, l'un des reproches majeurs faits aux théâtres de machines en Occident, reproche que Schreck ne peut ignorer : ils présentent des modèles souvent inefficaces du point de vue de l'économie moyens /effets obtenus ${ }^{25}$. Et il faut bien admettre que la sélection opérée par Schreck et Wang Zheng lève ce reproche, au moins en intention.

Mais encore, après avoir donné, à la suite de la préface, un glossaire des différentes parties des machines avec leurs noms sur lequel nous reviendrons, Wang Zheng présente trois tableaux analytiques : des agents et facteurs qui interviennent dans leur fonctionnement, de leurs capacités et enfin, des avantages qu'elles peuvent respectivement procurer.

- Les agents et facteurs utilisés dans les machines :

- les agents : la machine · les hommes (puissance humaine) · le cheval (puissance des chevaux) $\cdot$ le vent (puissance du vent) $\cdot$ l'eau (puissance de l'eau) · le vide (vide) · utilise le poids · utilise le levier · utilise la roue · utilise la vis à eau $\cdot$ utilise la vis · utilise la poutre d'une balance · utilise la poulie . utilise le cabestan ;

- les opérations : tourner $\cdot$ pousser $\cdot$ transporter $\cdot$ soulever $\cdot$ peser vers le bas . soulever vers le haut $\cdot$ porter;

- la puissance mise en œuvre : utilise peu de puissance - utilise une grande puissance $\cdot$ utilise une machine $\cdot$ utilise plusieurs machines $\cdot$ utilise des machines égales $\cdot$ utilise ce qui excède chaque autre machine $\cdot$ utilise des machines interconnectées · utilise des machines interdépendantes.

24. Préface, xu 3ab // Zhang et al., op. cit., vol. 2, p. 20.

25. "Ces machines pour la plupart ont été plus faciles à dessiner sur papier qu'à mettre en pratique " notera, après Salomon de Caus, Alessandro Capra ; voir Luiza Dolza, Hélène Vérin, 2004, op. cit., p. 17. 
- Les capacités de différentes machines : capable d'utiliser une petite puissance pour porter un grand poids - d'élever une chose pesante - de mouvoir un corps lourd sur une longue distance · de soulever successivement et sans fin une chose placée en bas - de faire qu'une chose inerte se meuve constamment - de faire qu'une chose silencieuse sonne automatiquement - de faire qu'une chose qui ne souffle pas souffle d'ellemême $\cdot$ de rendre une grande chose petite $\cdot$ de rendre une petite chose grande $\cdot$ de rendre une chose proche éloignée $\cdot$ de rendre proche une chose éloignée.

- Les avantages des différentes machines : économiser la grande puissance - éviter le grand travail - libérer de la servitude · éviter la catastrophe . économiser beaucoup · élargir la connaissance · augmenter la sagesse . rendre toutes les choses difficiles faciles sans danger.

Les éléments de cette analyse des effets propres aux machines se retrouvent tous dans les ouvrages sources, mais dispersés et à l'occasion de tel ou tel modèle. Au point que les historiens des théâtres de machines n'en ont pas fait l'examen systématique et se bornent en général à faire état du seul "obtenir plus d'effet avec moins de force ".

D’après l'historien Wang Bing, ces tableaux seraient directement empruntés à Matteo Ricci dans le Yi jihe yuanben yin, une introduction à la traduction des Éléments de géométrie parue en $1607^{26}$. Cette mention est importante. Elle signale l'effort d'intégration des différentes connaissances utiles à l'essor d'une mécanique chinoise et comme pour le QQTS, les adaptations auxquelles ce projet a donné lieu. Catherine Jami a montré comment cet effort s'inscrit dans le paysage intellectuel chinois.

Lorsque Xu Guangqi entreprend la traduction des Éléments avec Ricci, il a déjà été confronté en tant que fonctionnaire à des problèmes de mesure de terrains et de travaux d'irrigation, problèmes auxquels la géométrie s’applique de manière évidente. Il ressort de sa préface au Jihe yuanben qu'outre cette réponse qu’apporte l'ouvrage à ces préoccupations essentielles pour l'administration de l'empire, la grande force de celui-ci est la manière dont il est rédigé,

26. WANG Bing, «Ming-Qing shiqi (1610-1910) wulixue yizhu shumu kao » [A Textual Reasearch of the Bibliography of the Translated Works on Physics in the Ming and Qing Period (16101910)], Zhongguo keji shiliao, 1986, nº 7-5, p. 3-20. Notons qu'en Europe, à la même époque, les ingénieurs n’utilisent et ne se réfèrent qu’aux six premiers livres des Éléments d'Euclide. 
qui permet d'acquérir des certitudes quant aux résultats qu’on y obtient ${ }^{27}$.

On retrouve ici les préoccupations de Wang Zheng et en particulier la nécessité d'étayer ce qu'on avance, d'établir des connaissances fondées. Or les théâtres de machines qui lui servent de modèles ne répondent pas à cette exigence. D'où le recours aux traités préliminaires.

\section{Le contenu scientifique : les traités préliminaires}

En effet, si l'on considère les contraintes scientifiques qui déterminent les choix de dispositifs mécaniques et tout particulièrement les dimensions et les proportions retenues, les théâtres de machines, à très peu de choses près, n'en font pas état dans leurs explications ou « déclarations des figures ». Wang Zheng va rencontrer cette difficulté dans son projet de réaliser un livre de machines utile aux Chinois. La chose nous est rapportée dans sa préface, lorsqu'il raconte ses discussions avec Johann Schreck, Nicollo Longobardi et Adam Schall sur la traduction des explications de figures. Les rendre utilisables suppose que l'on sache déterminer ce qui n'y est pas indiqué : les dimensions des parties des machines, leurs raisons, leurs proportions et leur calcul.

Wang Zheng rapporte alors comment ses interlocuteurs l'ont persuadé de la nécessité de parcourir la suite des connaissances théoriques indispensables pour y parvenir. Il le fait, reprenant les propos de Schreck, en expliquant qu'en raison des dimensions, la mesure est apparue, et en raison des nombres, le calcul s'est développé. En raison de la mesure et du calcul la proportion idoine peut être trouvée, et en raison de la proportion, on acquiert la raison des choses qui permet à son tour d'établir la méthode, c'est-à-dire de choisir le type de dispositif approprié. Puis il résume : sans savoir la mesure et le calcul on ne peut certainement pas connaître la proportion et sans savoir la proportion on ne peut pas comprendre les diagrammes et explications des machines ${ }^{28}$.

27. Catherine JAMI, «Traductions et synthèses : Les Mathématiques occidentales en Chine, 16071782 ", in Marc Moyon, Marie-José Pestel et Martin Janvier (dir), Maths Express : Au carrefour des cultures, Paris, CIJM, 1986, p. 120-121.

28. Préface, 3a// Zhang et al.,op. cit., vol. 2, p. 18. 
Il faut rester attentif à ce texte et à la logique qui l'anime. Elle est insufflée à Wang Zheng par ses mentors qui sont des lettrés, eux-mêmes persuadés que la conception de machines est une application de la mécanique théorique. Le hiatus entre la logique d'exposition des théâtres de machines qui fascine Wang Zheng et celle qui procède des principes élémentaires de la mécanique du chapitre I du QQTS à l'examen des propriétés des machines simples du chapitre II, donne-t-il lieu à une explication ${ }^{29}$ ? Comment Wang Zheng en a-t-il pris acte?

Pour pouvoir répondre à cette question il faut se rapprocher du texte. Avant de s'y attacher on peut sans doute remarquer que c'est au niveau de l'économie générale du livre, avec ses trois chapitres successifs allant de l'abstrait au concret que s'exerce avec le plus de netteté l'introduction d'une logique étrangère au projet initial de Wang Zheng. Le résultat de la collaboration avec Schreck avec ses exigences propres qui est de rendre concevables ces modèles, conduit à la production d'un ouvrage qui réunit deux sortes d'écrits : le traité de mécanique et le théâtre de machines. Ces écrits, autonomes en Occident, expriment et utilisent différemment les catégories de la mécanique. Si l'on s'efforce de considérer ce qui fait l'unité de ces deux types de démarches, il s'agit bien de mettre à la disposition des lecteurs ce qui est nécessaire pour rendre effectifs les effets recherchés dans des mécanismes.

On peut donc dire que le QQTS est une invention de Johann Schreck et de ses collègues impliqués dans le projet lancé par Wang Zheng. Ils ont comblé l'un des manques reprochés aux théâtres de machines en exposant pédagogiquement des éléments de la science des mécaniques et des propriétés des machines simples. Ils ont fourni au lecteur chinois la possibilité de disposer dans un même volume des éléments théoriques nécessaires pour tirer parti des modèles présentés dans le chapitre III. Ils apportent même quelques données concernant les conditions pratiques de la fabrique comme lorsqu'ils indiquent les matériaux à utiliser, ou dans le chapitre II, en proposant des problèmes afin d'apprendre à mieux maîtriser le choix des méthodes. Tous facteurs sur lesquels les théâtres de machine restent à peu près muets, faisant fonds sur les savoirs ouvriers. Il en est de même pour Wang Zheng qui, dans sa préface, annonce que ses choix prennent en compte les capacités ouvrières disponibles. Il y revient à plusieurs reprises.

29. Chap. I: ZHANG et al., op. cit., vol. 2, p. 37-80; chap. II: vol. 2, p. 81-122. 


\section{La fabrique du chapitre III : un théâtre de machines chinois ?}

Nous abordons ici les difficultés propres à la lecture des théâtres de machines. Si l'on repart de l'hypothèse selon laquelle leur mode d'exposition vise à fournir au lecteur les moyens de comprendre comment le modèle fonctionne et de le reproduire, comment Wang Zheng va-t-il opérer sa transcription selon les codes décryptables par le lecteur chinois?

\section{La sinisation de la figuration graphique}

Si donc l'on considère la figuration comme un mode graphique d'explication des modèles, alors il faut que les moyens mis en ouvre dans les figures du QQTS aient, pour un Chinois, la même valeur informative. Dans les deux cas les moyens graphiques doivent être connus des lecteurs et obéir à des codes partagés. Quels sont ceux qui sont mis en œuvre dans les théâtres de machines et quels sont ceux qui sont à la disposition de Wang Zheng dans la culture graphique chinoise qui permettraient d'y transmettre l'information?

Notons tout d'abord un élément qui est repris sans changement notable et fonctionne parfaitement : les décors et les personnages. En effet, sur leurs planches gravées en pleines pages dans des décors naturels ou des ateliers, les machines sont animées par des personnages en action dont la présence et les attitudes sont importantes pour comprendre le fonctionnement de la machine. Wang Zheng ne s'y trompe pas, et ses personnages ont beau porter d'autres costumes et d'autres coiffures leur présence a la même efficacité que leurs modèles occidentaux. Néanmoins dans la présentation des machines et de leurs parties les théâtres utilisent des codes de représentation inconnus en Chine. En outre, ces codes sont multiples et s'interpénètrent. On en distinguera cinq : la perspective, avec les déformations locales de cette perspective, les arrachés, la mise en exergue d'éléments extraits de la machine, des représentations schématiques diverses de parties détachées.

\section{La perspective}

Les planches des théâtres de machines ne recourent pas à une perspective strictement géométrique mais plutôt à une scénographie qui permet 
simplement d'interpréter la place respective et relative des parties de la machine dans les trois dimensions. Pour ce faire, le rôle des ombres est important. Si la représentation globale obéit à une perspective, elle peut donner lieu localement à des déformations afin de faire mieux apparaître, donc comprendre, certains engrenages.

On peut ici faire état du débat sur le rôle de la perspective dans la constitution d'une sensibilité européenne éduquée à une perception de l'espace partagée à caractère géométrique, et qui aurait favorisé l'essor de la science moderne. Selon cette thèse, énoncée en 1952 par Panofsky, la représentation en perspective a mis sa marque dans l'esprit des hommes, l'a informé, de telle sorte qu'il leur devenait possible de concevoir les propriétés formelles de l'espace physique selon une géométrie démontrable. Partant, elle leur aurait permis de maîtriser techniquement leur action sur le monde ${ }^{30}$.

Dans «L'artiste de la Renaissance comme quantificateur » (1980), Samuel Y. Edgerton apportait une nouvelle pierre à la thèse de Panofsky ${ }^{31}$ en proposant d'insister "sur le caractère unique des révolutions scientifiques et artistiques de l'Europe occidentale ${ }^{32}$ ». Leur concomitance exprimant selon lui quelque changement fondamental et non spécifié dans l'expérience européenne de la perception du monde. Sans l'une, l'autre n'aurait pu apparaître. De sorte que selon Edgerton le nouveau langage pictural de la Renaissance fournit la capacité d'inventer des machines par le seul moyen du dessin ${ }^{33}$. Pour appuyer sa démonstration, il procédait à une étude comparative des dessins chinois reproduisant les figures de machines de Ramelli et manifestement incapables de les lire, il concluait que les Chinois ne pouvaient pas inventer de machines à l'aide du dessin seul parce qu'ils ne disposaient pas de la représentation en perspective. Dans la polémique

\footnotetext{
30. Erwin Panofsky, "Artist, Scientist, Genius: Notes on the Renaissance Dämmerung ", in The Renaissance: A Symposium, The Metropolitan Museum of Art, Feb. 8-10, New York, 1952, p. 77-93. Voir plus récemment Hans Belting, Florence et Bagdad, une histoire du regard entre Orient et Occident, Paris, Gallimard, 2012.

31. Samuel Y. Edgerton, "The Renaissance Artist as Quantifier ", in Margaret A. Hagen, The perception of Pictures, New York, Academic Press, 1980, t. I, p. 179-212.

32. "Instead of trying to discover elusive, one-on-one connections between individual geniuses of Renaissance art and seventeenth century science, we concentrate our investigations on shared uniqueness of the Western European scientific and artistic revolutions " : Samuel Y. EdGerton, op. cit., p. 181.

33. "The new Renaissance pictorial language allowed... the ability to invent machines solely by means of drawing ", art. cit., p. 210.
} 
relancée en 1985 par Michael Mahoney sur le sujet ${ }^{34}$, celui-ci ne se prive pas de souligner les a priori d'Edgerton et le caractère tautologique de son raisonnement, lorsqu'il conclut que " selon les standards occidentaux, il est difficile de comprendre comment la science et la technologie chinoise furent capables de quelque progrès, avec une aussi faible implication des artistes et de la peinture ». Où l'on voit, souligne Mahoney, que Edgerton fait de la représentation dessinée non plus un changement concomitant de l'innovation scientifique, mais un préalable ${ }^{35}$.

Si ce débat méritait d'être rappelé ici c'est surtout pour rendre compte de thèses qui ont tenté de penser l'émergence de la technologie occidentale en recourant à d'autres présupposés que ceux qui ne reconnaissent dans les machines qu'une application de la science des mécaniques. Dans ce débat, c'est la particularité de la conception technique et ses liens avec la figuration qui sont convoqués. Or, quels que soient les réponses apportées et les préjugés véhiculés, il se trouve précisément que le Chinois Wang Zheng, dans une tradition largement autochtone voyait dans la figuration graphique un mode d'intelligibilité des techniques mécaniques. La figure du treuil lulu (Fig. 1) extraite du Nong shu (Livre d'agriculture) (1313) de Wang Zhen illustre cette tradition, et montre bien que la représentation sans perspective de ce procédé d'irrigation permet d'en comprendre le principe de fonctionnement, lorsqu'elle est associée à son explication écrite :

Le treuil lulu. C'est une machine pour enrouler la corde d'un puits, avec un bois rond tournant et un bois, pour tirer l'eau. Au-dessus du puits on dresse un support pour un axe traversant un long moyeu à l'extrémité duquel on insère un bois courbe. Lorsqu’à l'aide de ses mains on fait tourner, la corde s'enroule sur le moyeu, ce qui fait monter le seau ${ }^{36}$.

\footnotetext{
34. Michael S. Mahoney, "Diagrams and Dynamics. Mathematical Perspectives on Edgerton's Thesis ", in John W. Shirley and F. David Hoeniger (ed.), Science and the Arts in the Renaissance, Washington, The Folger Shakespeare Library, 1985, p. 198-220. Version en ligne sur le site de l'université de Princeton. Nous citons cette version.

35. "In fact, by Western standards, it is hard to comprehend how Chinese science and technology was $(s i c)$ able to progress at all with so little involvement of artists or pictures » : ibid., p. 2.

36. WAng Yuhu (dir.), Wang Zhen Nong Shu (Livre d'agriculture de Wang Zhen), Beijing, Nongye chubanshe, 1981, 454 p., p. 339. Trad. auct.
} 


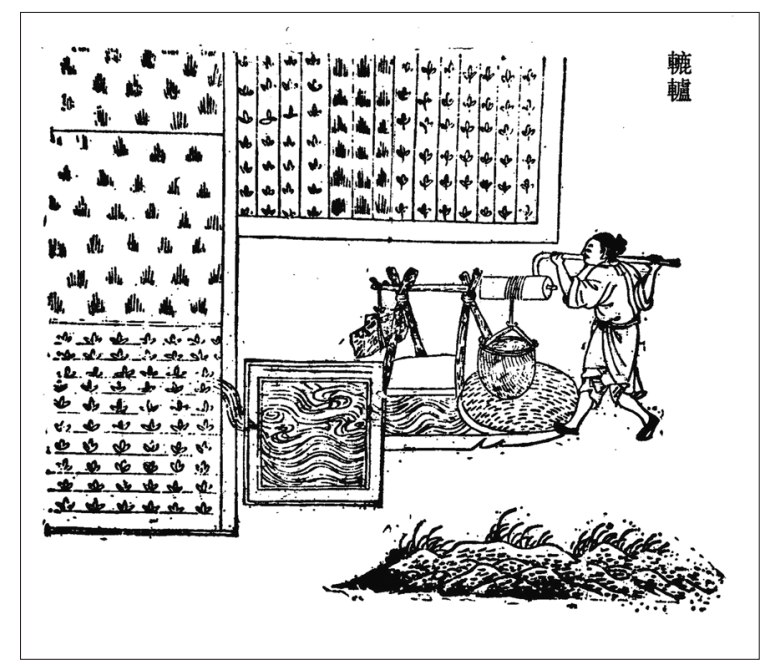

\section{Fig. 1. - Le treuil lulu}

« C'est une machine pour enrouler la corde d'un puits, avec un bois rond tournant et un bois pour tirer l'eau. Au-dessus du puits on dresse un support pour un axe traversant un long moyeu à l'extrémité duquel on insère un bois courbe. Lorsqu'à l'aide de ses mains on fait tourner, la corde s'enroule sur le moyeu, ce qui fait monter le seau. » Cette description associée à l'image permet la compréhension tant de la structure de l'engin que de sa fonction malgré l'absence de perspective dans le dessin. Fig. XX, Section 13 du Traité illustré des machines agricoles, troisième partie du Nong shu (1313) (Livre d’agriculture) de Wang Zhen.

\section{D’après Wang Yuhu, 1981, p. 339.}

Sans doute faut-il remarquer que cette représentation ressemble davantage aux schémas utilisés en Europe encore au XVII siècle par des mécaniciens ou par des ingénieurs, pour exposer au fabricant les caractéristiques du dispositif à réaliser. Il n'en demeure pas moins que la perspective - parfois bien étrange - utilisée dans les planches des théâtres de machines a plongé Wang Zheng dans un grand embarras, faisant de lui en quelque sorte, un précurseur d'Escher. C'est le cas de cette interprétation de la planche 9 du Machinae novae de Fausto Veranzio en QQTS, fig. 15, chap. 3, 44a ${ }^{37}$ (Fig. 2). 


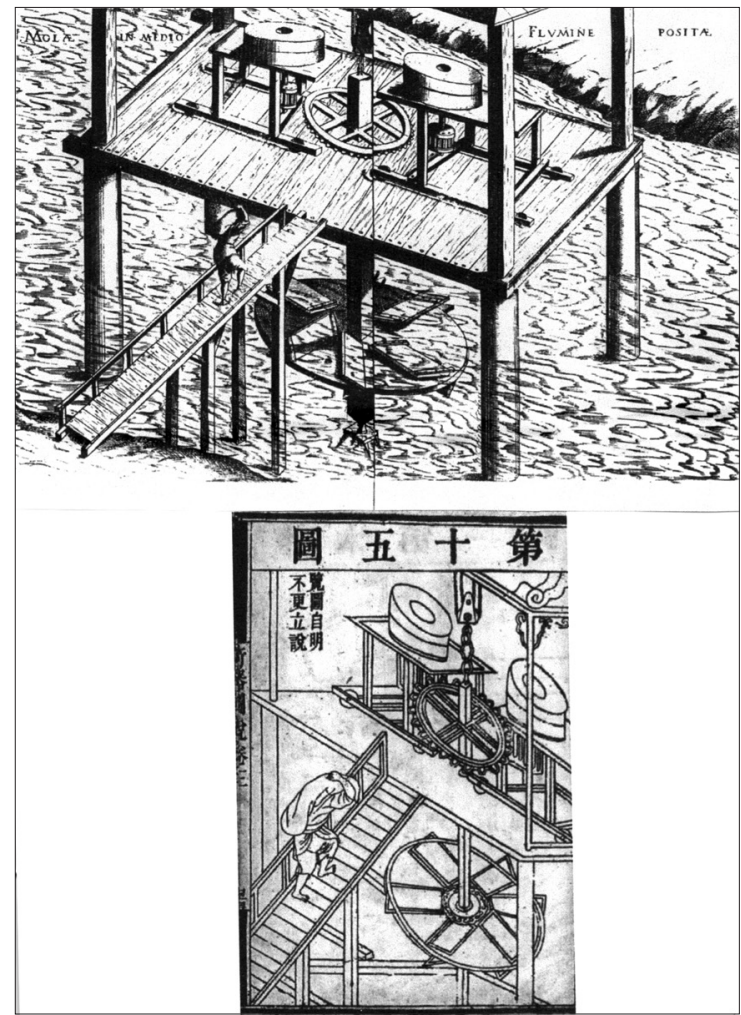

Fig. 2. - Fausto Veranzio, Machinae Novae, pl. IX. Wang Zheng, Qi qi Tu Shuo, III, pl. 5, p. 44a.

La planche de Veranzio est caractéristique des déformations imposées à la figuration d'une machine pour montrer des parties et des engrenages autrement peu visibles.

\section{L’arraché}

L'arraché est un autre code de représentation utilisé dans les théâtres de machines pour faire apparaître des parties intérieures qui autrement demeureraient cachées. Ce code est emprunté aux dessins d'architecture. Ramelli en fait grand usage. La technique utilisée dans le QQTS consiste à remplacer les bords déchiquetés de l'arraché par des nuées festonnées. Ce motif fréquent dans les dessins chinois peut-il acquérir un sens qui convienne particulièrement dans ce cas? Quoi qu'il en soit, dans le QQTS 
le résultat n'est pas très probant, même si l'on admet a priori qu'un regard averti d'autres codes pourrait en tirer parti (Fig. 3).

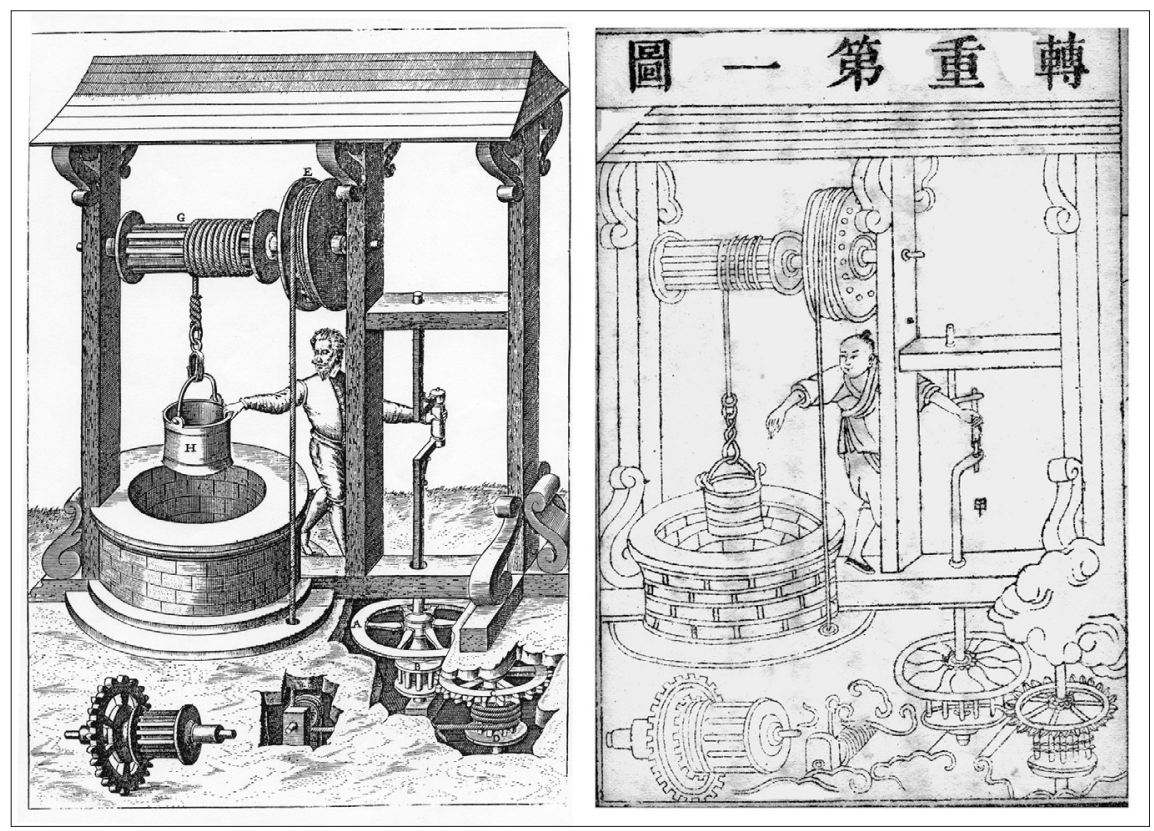

Fig. 3. - Agostino Ramelli, Le diverse et artificiose machine, pl. 85. - Wang Zheng, Qi qi Tu Shuo, III, pl. 1, p. 16b.

Interprétation de l'arraché comme code figurant les parties cachées de la machine.

\section{La mise en exergue d'éléments plus ou moins schématiques}

Très généralement, la reproduction de ces parties détachées et schématisées dont font usage certains théâtres de machines ne fait pas problème dans le QQTS : les mécanismes complexes sont redessinés efficacement. Nous retiendrons seulement ici l'exemple de ces pompes dont le développé, tiré de Zeising, est parfaitement reproduit (Fig. 4). 


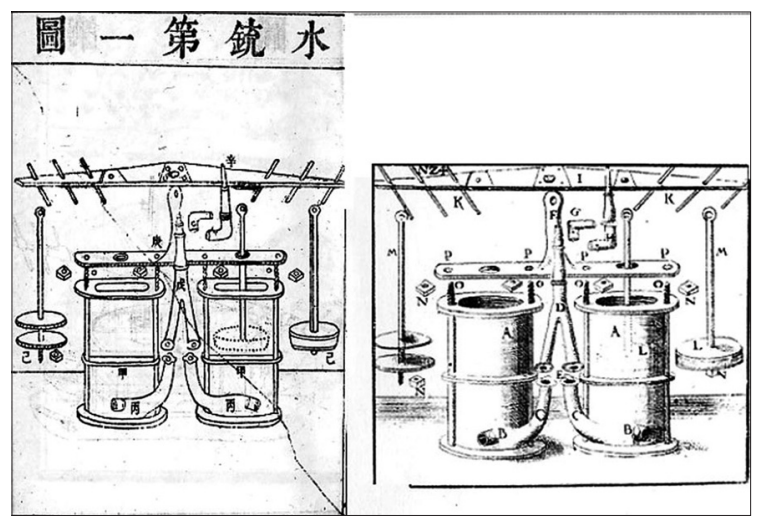

Fig. 4. - Heinrich Zeising, Theatri machinarum, II, pl. 24. - Wang Zheng, Qi qi Tu Shuo, III, pl. 1, p. 56a.

De tous les mécanismes en exergue repris dans le Qi qi Tu Shuo (juan 3, pl. 1, p. 56a), ce développé, technique de représentation encore rare en occident, est particulièrement réussi.

\section{La tradition graphique chinoise}

La pertinence de la figuration graphique chinoise, qui n'utilise pas la perspective mais obéit à ses propres codes dont on vient de voir des exemples, mériterait un examen particulier ${ }^{38}$. Nous nous bornerons à renvoyer à des figures tirées de livres beaucoup plus anciens et qui ont permis de transmettre des modèles de machines. L'une des plus complexes est certainement la tour-horloge astronomique à eau portant une sphère armillaire et un globe, conçue par Su Song (1020-1101) vers 1086 sur ordre impérial, et construite quatre années plus tard à Kaifeng, capitale de la dynastie des Song. En 1094, Su Song présenta au trône une monographie Xin yi xiang fa yao (Essentiel d'une nouvelle méthode pour sphère armillaire et globe céleste) qui nous est parvenue. Il y décrivait la structure et la fabrication de cette tour en particulier ${ }^{39}$.

38. Joseph Needham, Science and Civilisation in China, vol. rv, chap. 2, "Mechanical engineering", Cambridge, Cambridge University Press, 1965. Ce volume donne un large aperçu des réalisations chinoises dans le domaine de l'ingéniérie mécanique. Voir aussi Peter Golas, "Technological Illustration in China. A Post-Needham Perspective ", in Alain Arrault, Catherine Jami (dir.), Science and Technology in East Asia, Proceedings of the $\mathrm{XX}^{\text {th }}$ International Congress of History of Science (Liège, 20-26 July 1997), vol. Ix, Turnhout, Brepols, 2001, p. 43-58.

39. Joseph Needham, op. cit., p. 446-465, fait une analyse détaillée de cette machine complexe et en donne diverses représentations. Voir du même auteur, Le dialogue des civilisations, Chinel Occident, Paris, La Découverte, 1991, p. 141-152. 
On trouve dans le Nong shu (1313) de Wang Zhen les représentations de diverses sortes de meules et moulins accompagnées chacune d'une description. Nous choisirons deux modèles de meules multiples. La première sous l'intitulé "meules liées tournant ensemble " lian mo lian zhuan est ainsi décrite :

Pour la fabriquer, installer au centre une grande roue. La partie supérieure de son axe est assurée dans un châssis de bois, la partie inférieure repose dans un yijiu [manchon de métal-mortier]. On dispose ensuite sur le pourtour de la roue huit meules dont les dents de bois s'intercalent avec les rayons de la roue. Un buffle tire en tournant et ainsi les huit meules tournent en suivant les rayons de la roue. En utilisant peu de force, on voit beaucoup de résultats ${ }^{40}$.

Le dernier exemple est celui de " meules liées tournant avec l'eau "shui zhuan lian $m o^{41}$. L'auteur précise qu'il est nécessaire d'avoir un courant puissant pour actionner la roue qui, comme le montre l'image, doit entrầner neuf meules (Fig. 5).

Des moulins à eau du $x x^{\mathrm{e}}$ siècle ont révélé une structure analogue à celle de ce moulin du début du xive siècle.

Trois roues [dentées] sont rangées sur l'axe, chacune au contact d'une grande meule sur la périphérie de laquelle sont rangées des dents de bois. Les meules sont au-dessus de l'axe, soutenues par un plancher. Sur le côté de la meule on laisse une ouverture étroite pour laisser dépasser les rayons de la roue afin de frapper les dents de la meule au-dessus. Cette meule tourne et ses dents ensuite frappent celles des deux meules latérales. Ainsi, la force des trois roues entraîne neuf meules ${ }^{42}$.

\footnotetext{
40. WANG Yuhu, op. cit, p. 287. Trad. auct. La représentation de cette machine reprise du texte original se trouve dans Joseph NeedHam, op. cit., 1965, p. 196, fig. 451.

41. Ces "meules liées tournant avec l'eau ", sont représentées dans la section 14 du Traité illustré des machines agricoles, troisième partie du Nong shu (1313) (Livre d'agriculture) de WANG Zhen, in WANG Yuhu, op. cit, p. 355. Elles figurent également dans ZHANG et al., op. cit., vol. 1, p. 173, fig. 4-7.

42. WANG Yuhu, op. cit, p. 355. Trad auct.
} 


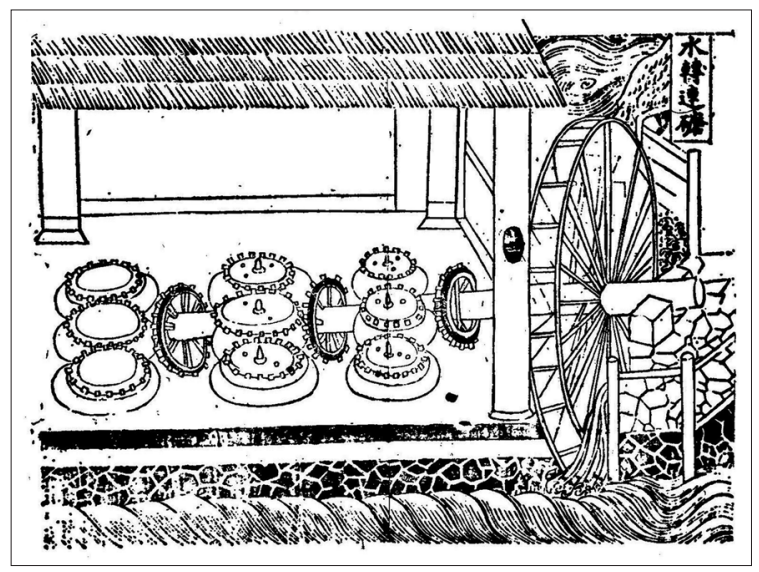

Fig. 5. - Section 14 du Traité illustré des machines agricoles, troisième partie du Nong shu (1313) (Livre d'agriculture) de Wang Zhen.

\section{D’après Wang Yuhu, 1981, p. 350.}

Même si, considérant l'image seule, on peut se poser la question de l'efficacité de telles machines en raison des forces de frottement, on constate que les descriptions sont claires et complètent l'image, la corrigeant même dans le second exemple. Ici, en effet, l'absence de représentation du plancher soutenant les meules, la non prise en compte de la position relative de ces dernières et de l'axe, la dimension trop grande des meules latérales pouvaient poser de sérieux problèmes de compréhension. Mais la lecture du texte permet de les résoudre. On peut aussi penser que pour les artisans qui construisaient des moulins, une image précise, à l'échelle, n'était pas essentielle.

Une preuve en est apportée dans une étude très détaillée ${ }^{43}$ qui permet la comparaison d'un modèle tiré à nouveau du Nong shu avec le relevé d'un moulin contemporain de structure analogue effectué sur le témoignage d'un ancien charpentier qui l'avait réparé une trentaine d'années plus tôt.

43. Zhang Baichun, "Zhongguo chuangtong shuilun ji qi quyu jixie", Ziran kexue shi yanjiu, $1994, n^{\circ} 13-2$, p. 155-163 et 1994, n 13-3, p. 254-253. (Les roues à eau traditionnelles chinoises et les machines qu'elles entraînent, Studies in the History of Natural Sciences). 


\section{L'explication textuelle}

Qu'est-ce qui, dans l'explication textuelle, rend compte de l'ambition déclarée de rendre intelligible et reproductible le modèle présenté ? En général, dans les théâtres de machines « l'explication de la figure » ou "déclaration de la figure " répond à l'ordre des opérations conceptuelles qui définissent un art, une technique : d'abord énoncer la fin recherchée, puis les avantages particuliers et les moyens mis en œuvre (moteur, machines simples). Mais ensuite les textes des différents théâtres sont loin d'être homogènes. Plus ou moins développés, ils ne procèdent pas tous selon la même logique d'exposition. On peut dégager en gros deux grandes méthodes : la première consiste à suivre l'ordre chronologique de la construction des dispositifs en y incluant quelques remarques d'ordre théorique ou fonctionnel. C'est souvent la méthode qu'utilise J. Besson. La deuxième consiste à en expliquer le fonctionnement en suivant le trajet du mouvement imprimé par le moteur dans les agencements successifs de machines simples qui assurent sa transmission et sa multiplication. C'est plutôt la méthode de Ramelli. Qu'est-ce que Wang Zheng va en faire ? Quelle méthode va-t-il suivre? Des textes, il ne dispose que d'une traduction verbale. En effet Wang Zheng connaît un peu de latin, grâce aux leçons de Nicolas Trigault, mais il doit ici recourir à la collaboration de Johann Schreck pour saisir le sens des explications qui accompagnent les figures. Si on confronte les textes sources à ceux qu'il propose, on s'aperçoit que si parfois il s'agit bien d'une traduction, dans la majorité des cas, comme il l'annonce lui-même dans sa préface, son commentaire est libre et il arrive qu'il soit complètement déconnecté de la source. Il procède à une description qui donne des indications de construction selon un ordre chronologique qui commence par le châssis qui supporte l'ensemble du dispositif. Ses explications ne deviennent fonctionnelles que pour certaines parties à propos desquelles il s'adonne à un luxe de détails, laissant dans un silence total d'autres parties importantes. C'est le cas de la machine à élever l'eau d'un puits, reprise de Ramelli (Voir Fig. 3). La fonctionnalité du dispositif est totalement perdue, mais encore, alors que Ramelli, pour décrire le système qui permet à l'homme de mettre en mouvement le seau se contente d'indiquer "manivelle faite en façon d'un vilebrequin » Wang Zheng fait de cette partie de la machine une description très développée. La combinaison figure-explication qu'il nous offre ne permet pas de comprendre comment le mouvement est transmis et démultiplié. 


\begin{tabular}{|l|l|}
\hline Ramelli, chapitre 85 & QQTS juan 3, p. 17a. \\
\hline «Ceste cy est une autre artificieuse façon de & «Tout d'abord, dans un po- \\
machine, moyennant laquelle un homme & teau dressé faire une manivelle \\
seul tirera l'eau d'un puis fort profond & comme indiqué en jia. Il faut \\
tres-facilement : d'autant que ledict homme & entre la partie supérieure et la \\
faisant par le moyen de la manivelle, qui & partie inférieure de l'axe instal- \\
est faite en façon d'un vilebrequin, tourner & ler symétriquement sur les côtés \\
la roue signée A, qui est mise sous terre, & perpendiculaires une 'branche' \\
ensemble avec la lanterne notée B, faict pa- & verticale que l'on fera tourner. \\
reillement en mesme temps tourner la roue & Afin de faciliter ce mouve- \\
dentée \& signée C, prenant ceste lanterne & ment on mettra autour de ce \\
avec ses fuseaux les dents de ladicte roue; & petit axe un tube de bois ou de \\
laquelle estant aussi soubs terre, \& ayant & bambou. On installe à lextré- \\
fiché dedans son arbre un tambourin noté & mité inférieure ou à l'extrémité \\
D, le faict semblablement tourner : lequel & supérieure une roue qui forme \\
tambourin entortillant sur soi la corde qui & le dispositif pour soulever des \\
passe sur la poulie F, \& qui sentortille par six & poids en tournant, ce que peut \\
ou sept tours à la roue qui est mise au-dessus & faire un seul homme. Aux deux \\
du puis notée E, la fait par ce moyen tour- & extrémités du poteau dressé on \\
ner ensemble avec la lanterne qui est fichée & dispose des manchons de fer \\
dedans son escieu signé G, laquelle lanterne & dans un mortier de fer fixé dans \\
par tels mouvemens entortillant sur soy la & la charpente; ainsi il tourne. \\
corde, laquelle avec un de ses bouts est atta- \\
chée au seau noté H, \& se tournant le tire en \\
hault au sommet du puis, comme clairement \\
on peut comprendre par son dessein ; \& & \\
voulant derechef tirer d’autre eau, on tour- & \\
nera la manivelle de l'autre costé, \& la corde & \\
de la susdicte lanterne se détortillant, le seau & \\
descendra au fond du puis, \& fera l'effect qui & \\
a esté dict cy-dessus. » & \\
\hline
\end{tabular}

Cet exemple invite à deux remarques. La première concerne bien sûr les savoirs et savoir-faire disponibles alors en Chine qui peuvent justifier le développement d'explications là où, en Europe, un mot suffit pour se faire comprendre ${ }^{44}$. La seconde remarque a trait aux libertés prises par Wang Zheng par rapport à la traduction orale des textes-sources par Schreck. Il a choisi de les transcrire plutôt que de les traduire littéralement dit-il dans sa préface, c'est-à-dire d'en rendre le sens communicable en chinois classique 
selon une logique propre. Il reste cependant une question sans réponse vraiment possible : la part que l'on doit à Wang Zheng dans la teneur de cet ouvrage.

Un premier élément de réponse nous est fourni par ce qui nous apparaît comme un souci terminologique. En effet, il fait figurer en tête du recueil après sa préface, une liste de soixante-six termes techniques qui sont sans doute les calques de termes trouvés dans les ouvrages sources. Il faut noter que cet ensemble ne regroupe pas tout le vocabulaire spécialisé qu'il va utiliser dans les descriptions des machines. On y trouve associés des termes repris du vocabulaire technique général. Il nous semble que la présentation à part de ce vocabulaire, fait très rare en chinois, est aussi la marque de l'influence que les textes européens ou Johann Schreck lui-même ont pu jouer dans la mise en forme de ce recueil. Cependant il faut se méfier des affirmations péremptoires en matière d'emprunt. Si nous considérons le tableau concernant la roue au tout début du chapitre II qui, d'une certaine manière, complète et éclaire la partie des termes relatifs à la roue d'un glossaire qui figure au tout début du premier chapitre, on peut y voir deux sources possibles qui peuvent ne pas être exclusives l'une de l'autre.

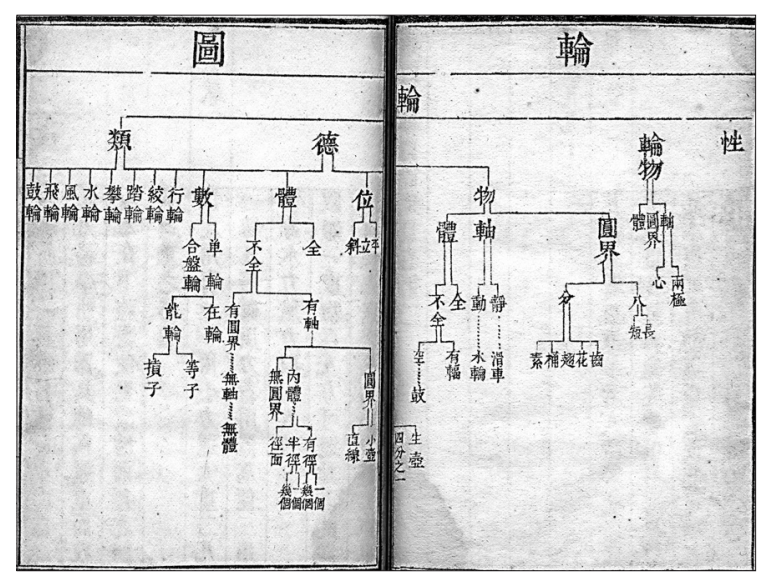

Fig. 6. - Diagramme relatif à la roue dans le Qi qi Tu Shuo, II, p. 1b-2a.

Lisant de droite à gauche, un sous-diagramme énonce d'abord les parties de la roue, axe avec moyeu et deux extrémités, bord et corps. Ensuite, c'est la nature de ces parties qui est indiquée, puis la position, horizontale, oblique, verticale ; reprise des diverses parties du corps; «nombre » : roue simple ou ensemble de roues, enfin, les diverses catégories, volant, tambour, roue à eau... 
Ce diagramme (Fig. 6) rappelle certainement des tableaux dichotomiques européens qui peuvent en être à la source ${ }^{45}$ mais une telle présentation schématique, de livres par exemple, était fréquente dans la littérature confucéenne. Nous renvoyons ici le lecteur à deux publications, l'étude par Michael Lackner (1996, p. 36-37) du « diagramme des voies et des vertus universelles ", dont l'auteur chinois présumé Wang Bo (1197-1274) cherche à élucider un passage du Zhongyong, d'une part, et la traduction d'une Étude de la sagesse en dix diagrammes d'un confucianiste coréen Yi Hwang (1501-1570) ${ }^{46}$.

Ce diagramme de la roue peut donc être plus justement perçu comme le résultat d'une synthèse entre deux approches voisines dans les deux cultures, un moyen peut-être aussi de faire percevoir au lecteur la proximité intellectuelle entre les lettrés de la Chine et ceux de l'Occident lointain.

À propos de la terminologie technique ${ }^{47}$ utilisée rappelons l'existence du glossaire sous forme de liste intitulé "Sommaire des noms de chacune des choses utilisées " Mei suo yong wu ming mu qui figure en préambule de l'ouvrage. Nous voudrions ici faire un bref rappel qui permet d'apprécier l'économie de la terminologie chinoise.

Chaque syllabe en chinois correspondant à un caractère de la langue écrite et, possédant un sens, il est très aisé de créer de nouveaux termes à partir de morphèmes monosyllabiques, soit par simple juxtaposition soit par détermination. Nous ne donnerons que quelques exemples. Pour la partie creuse fixe qui reçoit un axe mobile, dans le Livre d'agriculture de Wang Zhen (1313) on trouve le terme "manchon de métal-mortier " yijiu, tandis que notre auteur Wang Zheng choisit pour sa part «nid de fer » jiewo. Pour les néologismes, il traduit " cabestan " par " roue agitée " jiaolun, "volant " par " roue volante " feilun. S’il choisit de traduire " axe " par zhou dont

45. Systématiquement utilisés dans les ouvrages traitant de pratiques « réduites en art », Voir Hélène VÉRIN, "Rédiger et réduire en art : un projet de rationalisation des pratiques ", in Pascal Dubourg Glatigny et Hélène Vérin, Réduire en art, La technologie de la Renaissance aux Lumières, Paris, MSH, 2008, p. 44.

46. Michael LACKNER, « La position d'une expression dans un texte : explorations diagrammatiques de la signification ", Extrême-Occident, n ${ }^{\circ} 18,1996$, p. 35-49. YI Hwang, Étude de la sagesse en dix diagrammes, trad. fr., Paris, Éditions du Cerf, 2005, 136 p.

47. Pour une présentation de la terminologie relative à la mécanique, voir Iwo Amelung, « Weights and forces: the reception of western mechanics in the late imperial China ", in Michael LACKNER, Iwo Amelung and Joachim Kurtz (dir.), New Terms for New Ideas. WesternKnowledge and Lexical Change in Late Imperial China, Leyde, Brill, 2001, p. 197-232. 
le sens premier est l'axe reliant les roues d'un char, dans la description d'une meule il utilise un autre terme pour en nommer l'axe central vertical, shu qui désigne spécifiquement le pivot sur lequel est fixé un battant d'une porte. Enfin, dans le texte explicatif d'une machine à élever de l'eau de Besson (Besson 46), il nomme la partie supérieure de l'axe central par association de ces deux noms shuzhou littéralement "pivot-axe "; le mot utilisé dans l'édition de Besson de Genève de 1594 est "pivot ». Dans l'explication de la figure 3, Wang Zheng nomme l'axe portant la manivelle lizhu « poteau dressé ».

On peut supposer que si la compréhension du fonctionnement des machines pouvait poser des problèmes, c'est certainement plus par un manque d'explications précises ou par des erreurs dans l'interprétation des images que par manque d'un outil terminologique adéquat.

Pour conclure nous retiendrons donc qu'en 1627 les Chinois disposent d'un type d'ouvrage qui regroupe deux genres littéraires différents : le traité, qui enseigne et le théâtre qui dispose et fait voir la multiplicité des dispositifs mécaniques possibles. Cette réunion est intéressante parce qu'elle sollicite l'attention du lecteur en l'invitant à concevoir la machine comme une application des principes, maximes et règles de la mécanique. Elle fait apparaître ainsi un lecteur potentiel différent du lecteur européen, plus exigeant et réellement intéressé par la conception de machines. En effet, en Europe, les théâtres de machines sont destinés à des commanditaires, princes ou villes, à des protecteurs recherchés par les auteurs ou encore à des curieux ou amoureux des beaux livres à figures, qui ne sont pas concernés par la science des mécaniques. Quant aux traités, ils s'adressent à un tout autre public, celui des ingénieurs, des mathématiciens, des professeurs de mathématiques savantes ou pratiques qui enseignent leur discipline et la mécanique à de futurs ingénieurs et architectes, comme le fit Jacques Besson à Orléans. Jacques Besson dont les hésitations sur le contenu de son livre sont éclairantes pour notre propos. Cet auteur du premier théâtre de machine proposait dans sa version originale une introduction théorique, présente dans le manuscrit qui a été retrouvé au British Museum ${ }^{48}$. Mais il

48. British museum, ms add. 17921. Voir Alexander G. KeLLER, «A manuscript version of Jacques Besson's Book of Machines, with His Unpublished Principles of Mechanics ", in Bert S. Hall et Derno C. West (dir.), On Pre-Modern Technology and Science, a volume in honor of Lynn White jr., Malibu, 1976, p. 75-103. 
a renoncé à l'intégrer dans son Livre premier des instruments mathématiques et méchaniques, première version publiée de son Théâtre de machines.

Le Qi Qi Tu Shuo avec sa juxtaposition de traités et d'un " théâtre " est ainsi une figure singulière de l'état des savoirs mécaniques au début du $\mathrm{XVII}^{\mathrm{e}}$ siècle, et en même temps l'indice d'un effort de collaboration maximum de Johann Schreck pour rendre réalisable le projet de Wang Zheng d'un ouvrage utile à la Chine.

\section{Auteurs}

Georges Métailié est membre honoraire du Centre Alexandre-Koyré. Ses recherches portent principalement sur l'histoire des savoirs naturalistes en Chine et au Japon. Il a publié avec Francine Fèvre, Dictionnaire Ricci des plantes de Chine, chinois - français, latin, anglais, Paris, Association Ricci/Cerf, 2005, et "Traditional botany: An ethnobotanical approach », in Joseph Needham, Science and Civilisation in China, vol. 6, part. 4, Cambridge, Cambridge University Press, 2015. Contact : gsmetailie@orange.fr

Docteur d'État en philosophie, Hélène Vérin a été chercheuse au CNRS en économie, philosophie puis histoire. Ses recherches portent sur l'histoire et la philosophie des techniques et des sciences de l'ingénieur à l'époque moderne. Elle a publié en collaboration avec Pascal Dubourg Glatigny, Réduire en art, la technologie de la Renaissance aux Lumières, Paris, MSH, 2008, et réédité Entrepreneurs, entreprise. Histoire d'une idée, Paris, Classiques Garnier, 2011. Contact : verin. helene@me.com 